\title{
Sergio Tonatiuh Serrano Hernández, La golosina del oro. La producción de metales preciosos en San Luis Potosi y su circulación global en mercados orientales y occidentales durante el siglo XVII, San Luis Potosí, El Colegio de San Luis/El Colegio de Michoacán/Instituto de Investigaciones Dr. José María Luis Mora, 2018.
}

\author{
José Manuel Díaz Blanco ${ }^{1,}$ * (D) 0000-0002-5250-701X
}

\footnotetext{
${ }^{1}$ Universidad de Sevilla

*Correspondencia: diazblanco@us.es
}

En cierta ocasión, Adriano Prosperi escribió "Con l'oblio lo storico ha un conflitto professionale". Es difícil eludir esta máxima al leer que "la historia de [el cerro de] San Pedro es la del olvido”, y que ese olvido "es hoy una herida en la conciencia colectiva de México". ¿Puede un libro de historia curar y cicatrizar un daño asî? Sergio Serrano Hernández no se arredró ante una posible respuesta negativa; asumió el conflicto profesional del historiador y desarrolló durante años, entre México y España, una investigación inspirada por la conciencia ciudadana, el amor hacia la tierra de San Luis y su pasado, así como la curiosidad intelectual por indagar por qué su estudio podía contribuir al esclarecimiento de cuestiones y debates fundamentales en torno a la economía global del siglo xvII. Prosperi concluía, sobre aquel combate de la memoria, que al final "i suoi prodotti migliori sono stati raggiunti quando le genealogie ufficiali del presente sono state sovvertite dallaffiorare inquietante di qualcosa di dimenticato", : 1

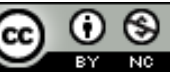

Esta obra está protegida bajo una Licencia Creative Commons Atribución-NoComercial 4.0 Internacional

${ }^{1}$ Adriano Prosperi, L'eresia del Libro Grande. Storia di Giorgio Siculo e della sua setta, Milán, Feltrinelli, 2011 , p. 7.

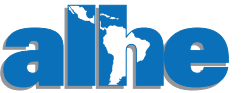

http://alhe.mora.edu.mx
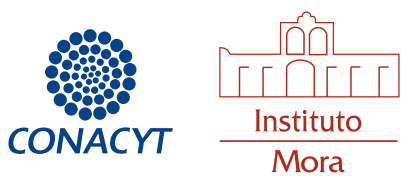
La investigación comienza en un marco geográfico, aparentemente circunscrito, dentro de una escala local-regional entre San Luis Potosí, el conjunto de su alcaldía mayor y el cerro minero de San Pedro. El espacio formaba parte del arco chichimeca o, como prefiere el autor, del septentrión novohispano, que se incorporó al virreinato de México durante la segunda mitad del siglo XVI, y se configuró como una frontera en permanente mutación. Serrano empezó por imaginar ese territorio como un paisaje social dominado por la actividad agrícola, influido por un escenario historiográfico donde las cuestiones mineras no siempre se valoraban lo suficiente frente a la actividad agraria, salvo en casos especialmente rotundos como Zacatecas. Sin embargo, el trabajo en el archivo no tardó en desengañarlo y demostrarle algo muy diferente. Le reveló, pues, que la minería era el verdadero motor económico de la región y que los demás sectores se articularon en torno a sus necesidades y sus impulsos. Definitivamente, aquella sería una investigación sobre minas y metales preciosos.

Tras haber confrontado sus propios apriorismos, Serrano se posicionó ante el trabajo y los enfoques de la historiografía previa sobre San Luis. Tal como la presenta el propio autor, se trataba de una historiografía escueta, pero valiosa. Su referencia clásica recaía en la Historia de San Luis Potosí (1946) de Primo Feliciano Velázquez y en años recientes se había enriquecido gracias al esfuerzo de investigadores formados en las licenciaturas y maestrías en historia abiertas en la ciudad desde la década de 1990. Esta bibliografía, al igual que otras mucho más pobladas, dejaba silencios e interrogantes. Quedaban muchos recovecos de la cultura minera pendientes aún de escudriñar. Más aún, sobrevolaba una duda que requería alguna solución. La cuestión se remontaba a la obra de Velázquez y suponía que la extracción de metales preciosos había entrado en una larga fase depresiva durante el primer cuarto del siglo xvir, arrastrando consigo al resto de la economía regional. La mayoría de los autores posteriores aprobaron y reprodujeron este esquema, aunque alguna voz aislada apuntara la posibilidad de que el Seiscientos hubiera sido de otra manera distinta. Como veremos a continuación, esta opción alternativa fue desarrollada por Serrano hasta diferenciarlo del tronco principal de la historiografía potosina.

La clave para consolidar esta opción consistía, precisamente, en pensar más allá de lo local y lo regional. Es entonces cuando el libro despega. Las razones no son difíciles de suponer. Los avatares de San Luis Potosí daban muestras de convertirse en un excelente laboratorio desde el cual generar argumentos útiles para el debate de la crisis del siglo Xvi en América. No hace falta recordar que la problemática suscitó una de las controversias más encendidas entre los modernistas del siglo $\mathrm{xx}$, desde que surgiera para explicar la evolución económica europea durante aquella centuria, ni que su recepción por parte de la historiografía latinoamericana tampoco se vio exenta de disensos. Hubo posiciones que abogaban por reproducir con convicción la postura europea del asunto, y hubo posturas contrarias que negaban esta posibilidad y propugnaban un siglo xvir de desarrollo económico y social en Hispanoamérica, auténtica contracoyuntura atlántica, tal como propusiera Ruggiero Romano.

Una de las investigaciones más significativas para determinar la cuestión de la crisis del siglo XVII vino de la mano de Herbert S. Klein y John J. TePaske. Como es bien sabido, ambos coordinaron una investigación masiva que registró sistemáticamente los ingresos de las cajas de la Real Hacienda en Indias. Los resultados hablan, en primer término, de rendimientos tributarios, materia que por sí misma ya resulta de importancia capital. No obstante, dada la matriz exactora de la fiscalidad, la información se convertió en una puerta abierta (a veces la mejor, ocasionalmente la única) al conocimiento de la producción y el intercambio comercial; al conjunto de la economía, y en particular al de la minería, que en muchas regiones de América actuaba como el impulsor 
principal y la fuente de ingresos más jugosa. Desde entonces, varias obras han asumido esa perspectiva fiscal como vía de acceso al desarrollo económico y la determinación de conclusiones en torno a la crisis del siglo XVII, algunas tan reconocidas como la de Kenneth Andrien.

Sergio Serrano comprendió perfectamente el potencial de esta perspectiva clásica. Era justo el elemento que necesitaba para profundizar en la historia de San Luis y encajarla en un marco más general. Tras obtener resultados insuficientes en el Archivo Histórico de San Luis y en el General de la Nación de México, encontró el material idóneo en el Archivo General de Indias de Sevilla. Lo halló entre los mismos fondos de Contaduría que investigaron Klein y TePaske, sin embargo se acercó a ellos definiendo una estrategia de investigación diferente. Los estadunidenses habían planteado un trabajo extensivo, que cubría una geografía enorme y que, por tanto, no podía profundizar en los detalles de ningún sitio en particular. Les bastó con recolectar los datos consignados en los sumarios de cargo y data de los oficiales reales.

Serrano, a diferencia de ellos, pretendía una reconstrucción detallada de San Luis, por lo que necesitaba de una investigación más intensiva. Analizó las fuentes, las cuestionó y comprendió el sistema fiscal y administrativo español, que empezaba en los reales de minas y acababa en la Contaduría del Consejo de Indias. A lo largo de la extensa y completa explicación de dicho sistema, que ocupa el capítulo 2, Serrano presenta un par de conclusiones esenciales para el desarrollo de su proyecto: los sumarios (o cartas cuentas) eran un compendio final de la información generada por la fiscalización de la actividad económica y, por tanto, si se trazaba la genealogía de la documentación, se podía remontar el flujo de los datos a fases precedentes, en las que se habían registrado de manera más desagregada. Las cartas cuentas procedían de los libros mayores de la caja y estos, a su vez, de otros libros comunes, particulares o manuales. Presumiblemente, la información más amplia se encontraba en estos últimos, elaborados pacientemente por los oficiales de las cajas. Pero los de la oficina de San Luis se hallan perdidos, acaso destruidos para siempre. Serrano solo pudo localizar ocho libros comunes en el Archivo de San Luis. Sin embargo, los libros mayores, preparados por los oficiales del Tribunal Mayor de Cuentas, sí se conservan. Pese a pequeñas lagunas y extravíos, los fondos del Archivo de Indias permitían seguir el curso completo del siglo Xvir.

El esfuerzo de una lectura sistemática permitió a Sergio Serrano registrar todos los datos relevantes de los libros mayores. Los ordenó dentro de una base de datos elaborada con el programa FileMaker, a la que denominó Sistema de Información Histórica (SIH), y los relacionó para construir una visión estructural y coyuntural de la minería potosina que se expone detalladamente en el capítulo 3 del libro. Este tercer capítulo, denominado "La producción minera de San Luis Potosí durante el siglo xviı”, constituye el núcleo conceptual de la obra. Su lectura no resulta sencilla, debe reconocerse, pero el esfuerzo bien merece la pena. Tras la aridez del lenguaje matemático se descubre la ambiciosa aventura intelectual de reconstruir los datos más menudos, los cotidianos, que se perdieron con los libros de ensaye, y la voluntad de, al menos, aproximarse a cómo debieron de ser. Los resultados abren una perspectiva novedosa en el análisis de la minería colonial: la posibilidad de agregar datos mensual y trimestralmente. Hasta la fecha la historiografía solo había trabajado con agregaciones anuales. Estas sirven para plantear patrones de evolución al largo plazo (usualmente visiones seculares), pero aportan poco o nada a la determinación de otras cuestiones como la estacionalidad del trabajo en los reales de minas. El propio autor reconoce la fragilidad de la técnica y, por tanto, de las impresiones que puedan construirse a través de su aplicación. Sin embargo, cabe también convenir que se trata de un paso adelante en la comprensión de esta actividad económica fundamental y que el método debería aplicarse a los grandes centros del XVII como Potosí y Zacatecas, a fin de calibrar su verdadera utilidad y alcance. 
La agregación anual puede parecer más definida, y lo es, ciertamente, en cuanto se refiere a la ordenación y exposición de los datos. No obstante, su interpretación está sujeta a una variedad de análisis que Serrano esgrime polémicamente frente a la historiografía de San Luis. Las series de producción de todo el siglo (inferidas en los primeros años de la caja de México y, desde 1628, de la del propio lugar) parecen avalar con escasas dudas la decadencia de la minería a lo largo del siglo XVII. La pendiente presenta un marcado perfil decreciente en los gráficos 63-67, en los que las agregaciones quinquenales se suman a las anuales. Cualquiera se habría sumado sin demasiados escrúpulos a las conclusiones comunes desde la época de Primo Feliciano Velázquez. Sin embargo, Serrano demuestra que sí hay lugar para la discrepancia. La visión alternativa que propone incluye importantes sugerencias entre las que cabe recordar: 1) una expansión del ciclo positivo hasta mediados de los años 30; 2) una disminución del ciclo contractivo hasta el decenio de los 60, y 3) una relativización del estancamiento de las últimas décadas desde la valoración de la producción bimetálica de las minas de San Luis. En efecto, San Luis era una cierta excepción en el panorama minero novohispano, por no decir americano. Sus reales se contaban entre los pocos que presentaban una producción significativa de oro, cuyo valor era, no es preciso recordarlo, muy superior al de la plata. Ante esta realidad, Serrano argumenta que las empresas potosinas se centraron en la calidad antes que en la cantidad, produjeron poco deliberadamente, ahorraron costes de producción y maximizaron la rentabilidad merced al alto valor en el mercado de los mejores metales. Si a ello se añade una cuarta consideración (casi una sugerencia, pues el tema se encuentra menos presente en la investigación), que avisa de que a lo largo del siglo XvII la economía regional dependió menos de la minería y se benefició del desarrollo de otros sectores, nos encontramos con un repertorio de proposiciones que, efectivamente, invitan a matizar y discutir sobre la panorámica de un siglo completo que solo pueda entenderse en términos de recesión económica.

Una vez aquí, había elementos suficientes con los que esbozar una propuesta sobre de la crisis del siglo XVII. De hecho, la versión original de la obra (que circuló bastante tiempo en internet) pasaba directamente de este capítulo al apartado de conclusiones. Sin embargo, aprovechando el retraso que sufrió la impresión, Serrano incorporó un nuevo capítulo. El añadido es un gran acierto. La concepción original de la investigación atendía fundamentalmente los niveles de producción de metales preciosos. El enfoque macro predominaba, igual que el análisis cuantitativo, y las cuestiones en juego -la crisis del XVII-, si bien mantenían su vigencia, engarzaban con preocupaciones que llevaban décadas en la agenda historiográfica. Serrano asumió que, en estos tiempos en los que la historia global se impone como prioridad investigadora, la oportunidad de abordar la presencia de los metales de San Luis en los circuitos de la economía mundial era más que interesante.

Nuevamente, la obra ofrece bastante al lector. Mientras los estudios sobre la primera globalización suelen preferir las rutas oceánicas (y se plantea cómo la historia global se construye sobre el ejercicio de la historia marítima), su análisis comienza con la reconstrucción de los intercambios y los caminos terrestres que conectaban la alcaldía mayor de San Luis con la Ciudad de México. La capital del virreinato era el primer destino de buena parte de la plata potosina, circunstancia que a principios de siglo venía potenciada por la inexistencia de la caja propia y la carencia de medios técnicos para separar el oro de la plata en el lugar de producción. El registro efectuado en San Luis de los metales llevados a México quedó reflejado en una serie documental designada "manifestaciones de platas", conservada hoy en el Archivo Histórico del Estado de San Luis Potosí. Serrano ya había aprovechado la fuente en un interesante trabajo, publicado en un libro coordinado por 
Guillermina del Valle y Antonio Ibarra ${ }^{2}$ Los dos textos se vinculan y complementan. Este capítulo aportado al libro coordinado por Del Valle e Ibarra es una radiografía de ese mercado de metales siguiendo la técnica del análisis de redes sociales (ARS). El capítulo de La golosina del oro, en cambio, optaba por catalogar a los protagonistas de dicho mercado, tanto a los mineros potosinos como a los mercaderes mexicanos, según el volumen de metal con el que operaban. El resultado es una interesante historia social, en la que los individuos concretos con sus nombres propios aparecen con mayor fruición que en los primeros capítulos del libro. Tal identificación es fundamental, porque a continuación puede comprobarse cómo los receptores mexicanos empleaban esa plata (y la que les llegaba de otros sitios, claro está) en los mercados globales. Podían destinarla a Asia, a través de Acapulco, o podían remitirla a Europa, vía Veracruz, bien hacia Manila o bien hacia Sevilla. La plata de San Luis llegó así a la misma ciudad donde se custodian los papeles de su real caja y en la que Serrano Hernández pensó y preparó buena parte de su obra.

Las "Consideraciones finales" recopilan varias de las cuestiones fundamentales aparecidas a lo largo del texto y las emplean como premisas para preguntas nuevas. Por ejemplo, ¿qué lugar ocupaba San Luis entre los centros mineros de la Nueva España? Los datos recabados permiten afirmar que, como mínimo, le correspondía el tercer puesto entre los focos productores de plata y que, probablemente, escalaba hasta el segundo. Zacatecas era la referencia principal, no hay duda. A continuación, venía México, pero como sus datos se encuentran inflados por las remisiones que le llegaban de cajas subsidiarias, lo más seguro es que sus registros propios sean inferiores a los de San Luis. Solo esto bastaría para tener en gran consideración la minería potosina. Sin embargo, la verdadera talla de sus reales se desvela al considerar la producción de oro. Producían casi el 87 \% de todo el oro novohispano. San Luis dominaba la producción áurea, incluso más que Zacatecas la argéntea. De hecho, en el siglo XviI solo cedía ante la producción registrada en Popayán, Nueva Granada. Ése era su verdadero rasgo característico y diferenciador. Una de las máximas aportaciones de la obra es calibrar y reivindicar esta circunstancia, hasta ahora escasamente considerada, por no decir ignorada.

Era algo olvidado. Pues la historia de San Pedro era la del olvido. Ahora ya lo es menos, un poco menos, y se lo debemos a Sergio Serrano, quien aceptó el conflicto profesional del historiador y subvirtió las genealogías oficiales del presente con el aflorar inquietante de algo cancelado en la memoria. Los hallazgos sobre San Luis y la minería novohispana derivaron en la oportunidad o necesidad de revisitar la crisis del siglo XviI. Serrano la satisfizo en un importante artículo publicado junto a Herbert Klein en 2019 (complemento o continuación de otro publicado en 1981 por el propio Klein junto a John J. TePaske), que finaliza rotundamente: "Thus, to answer the question of was there a generalised $17^{\text {th }}$ century crisis in Spanish America, the answer appears to be no" ? Otras incógnitas están siendo abordadas en una investigación sobre las cajas reales de Indias en el siglo XVII, que actualmente entra en su fase final. Sergio Serrano no detiene su labor. La golosina del conocimiento es casi tan dulce como la del oro.

\footnotetext{
${ }^{2}$ Sergio Serrano Hernández, "Mercado de minerales y redes comerciales en San Luis Potosí a comienzos del siglo XVII”, en Guillermina del Valle Pavón y Antonio Ibarra (coords.), Redes, corporaciones comerciales y mercados hispanoamericanos en la economía global, siglos XVII-XIX, México, Instituto Mora, 2017, pp. 27-68.

${ }^{3}$ Herbert Klein y Sergio Serrano, "Was there a 17th century crisis in Spanish America?”, Revista de Historia Económica, 37-1 (2019), pp. 43-80. El otro artículo mencionado es Herbert Klein y John J. TePaske, "The Seventeenth-Century Crisis in New Spain: Myth or Reality?", Past and Present, 90 (1981), pp. 116-135.
} 